\title{
Strates
}

STRATES Matériaux pour la recherche en sciences sociales

$4 \mid 1989$

Dossier : images réfléchies. Paroles d'un paysan révolutionnaire

\section{Repères pour l'analyse de cinquante ans d'écologie et de politiques d'environnement}

\section{Marie Claude Guerrini}

\section{(2) OpenEdition}

Journals

Édition électronique

URL : http://journals.openedition.org/strates/4652

DOI : $10.4000 /$ strates.4652

ISSN : $1777-5442$

Éditeur

Laboratoire Ladyss

Édition imprimée

Date de publication : 31 décembre 1989

ISSN : 0768-8067

Référence électronique

Marie Claude Guerrini, «Repères pour l'analyse de cinquante ans d'écologie et de politiques

d'environnement », Strates [En ligne], 4| 1989, mis en ligne le 19 mai 2008, consulté le 08 septembre 2020. URL : http://journals.openedition.org/strates/4652 ; DOI : https://doi.org/10.4000/strates.4652

Ce document a été généré automatiquement le 8 septembre 2020

Tous droits réservés 


\title{
Repères pour l'analyse de cinquante ans d'écologie et de politiques d'environnement
}

\author{
Marie Claude Guerrini
}

1 Ce tableau synoptique s'inscrit dans le travail, engagé par l'équipe Systèmes de pouvoir et Dynamiques des Espaces ruraux du Laboratoire avec les équipes espagnole et polonaise, sur une analyse comparée des politiques d'Aménagement rural et d'Environnement ${ }^{1}$.

2 Pour l'Environnement, nous ne pouvions adopter exactement le même schéma d'analyse que pour l'Aménagement rural. ${ }^{2}$

3 Si l'Ecologie est une science, ${ }^{3}$ le concept d'environnement ${ }^{4}$ est multiforme et l'analyse des politiques le concernant se doit d'aborder de nombreux problèmes. Pour beaucoup, lorsque l'on parle de l'environnement, il s'agit avant tout de protéger la nature présente dans la plupart des compartiments de la vie sociale (de l'eau aux arbres, du sol aux animaux, ou encore de l'air respiré au cadre de vie) contre les agressions associées au développement des sociétés industrielles, consommatrices de davantage de biens et donc émettrices d'autant de déchets et de polluants.

4 Bien que l'idée de protection ne soit pas tout à fait nouvelle, on ne constate pas un continuum absolu. Il faut signaler au $\mathrm{XIX}^{\mathrm{e}}$ siècle la promulgation de quelques lois destinées à protéger le patrimoine ou qui, prenant en compte les préoccupations des hygiénistes, visent à réglementer les activités polluantes. Parmi les plus importantes, il faut citer ici : la loi de 1913 sur les Monuments Historiques, celle de 1917 sur les établissements dangereux, insalubres et incommodes et celle de 1930 sur la protection des Monuments Naturels et des Sites.

5 L'élaboration d'une politique de l'Environnement est récente. Ainsi, l'intrusion de la notion, très forte, de protection, de sauvegarde, de conservation des ressources existantes, dans tous les compartiments de l'aménagement du territoire, aussi bien dans l'espace rural que dans l'espace urbain, émerge après la seconde guerre mondiale, et se concrétise surtout dans la deuxième moitié de ce siècle. Cette politique se situe après la structuration de l'écologie scientifique entre 1935 et 1955 dont nous indiquons 
les principales publications. Nous avons donc volontairement commencé la chronologie de la politique de l'environnement après la seconde guerre mondiale. Centrée sur les décennies 1960 et 1970, elle correspond à l'émergence d'une prise de conscience publique des problèmes d'environnement au début des années soixante; une accélération de la sensibilité écologique se produit à la fin de la décennie, ceci se traduisant en particulier dans les décisions politiques du gouvernement par la création, en 1971, du premier ministère français de l'Environnement.

6 Nous n'avons pas considéré l'environnement uniquement sous un angle administrativo-juridique. Il était en effet important de mettre en parallèle le cadre juridique avec les événements scientifiques ou médiatiques, les catastrophes écologiques et les attitudes ou prises de position au niveau national ou international qui ont précédé, accompagné ou suivi les actes «politiques» du gouvernement français.

7 Ce tableau ne prétend pas être exhaustif, et veut simplement poser des jalons qui permettront une analyse plus fine de tel ou tel aspect de la bibliographie ou des politiques. Nous présentons des données issues de sources différentes, inégalement accessibles, ou parmi lesquelles nous avons parfois opéré un tri. Dans la «jungle » que représente la législation qui prend en compte l'environnement, nous avons retenu les textes législatifs qui manifestent le souci du maintien des ressources. La prise de conscience de la fragilité des milieux et de la responsabilité des sociétés dans leur gestion a entraîné une multiplication des prises de positions médiatiques ou militantes. On assiste ainsi à un développement très important des associations soucieuses de préserver le cadre de vie. Ne pouvant les citer toutes ${ }^{5},{ }^{6}$ nous avons retenu celles qui ont un rang national, et, dans la mesure du possible, celles qui se situent à un niveau régional ou gèrent des Réserves Naturelles. La bibliographie concernant aussi bien les ouvrages scientifiques que ceux de vulgarisation ou d'information a été faite d'après les connaissances de l'auteur et grâce à la consultation de la bibliographie d'ouvrages de référence. Enfin, nous n'avons signalé que les accidents ou catastrophes technologiques. Les catastrophes naturelles n'ont pratiquement pas été recensées, sauf sous l'aspect législatif du risque, et l'analyse de leur concordance avec le développement de certaines activités reste à faire.

\section{NOTES}

1. Ce travail s'inscrit aussi dans une réflexion sur les conceptions de la nature et des milieux depuis la Renaissance menée par Y. LUGINBUHL et M.C. ROBIC de la même équipe.

2. Cf. Y.LUGINBUHL, Chronologie de création de procédures, organismes et institutions concernées par l'Aménagement Rural, STRATES, 1.

3. Ce néologisme défini par E. HAECKEL en 1866 comme science qui étudie les relations des êtres vivants avec leur milieu s'est structuré dans un premier temps grâce aux 
travaux de Schimper et de Waming au tournant $\mathrm{du} x \mathrm{x}^{\mathrm{e}}$ siècle et s'est développé sous sa forme systémique dans les années 30 (cf. P. ACOT, 1988).

4. « Ensemble de conditions naturelles et culturelles susceptibles d'agir sur les organismes vivants et les activités humaines » (Dic. Robert, 1984), ou encore « Ensemble des éléments objectifs et subjectifs constituant le cadre de vie d'un individu » (Grand Larousse 1987).

5. Il existe actuellement plus de 5000 associations en France se réclamant de la défense de l'environnement stricto sensu.

6. Dans le tableau, les publications marquées $(*)$ correspondent aux revues publiées par des associations.

\section{RÉSUMÉS}

On présente ici dans un tableau synoptique les jalons pour une analyse d'un demi-siècle de politiques d'environnement. Les axes législatifs français et les organismes et structures mis en place pour leur application sont considérés. Il a paru intéressant, pour élargir le champ de l'analyse, de mettre en regard le développement du mouvement écologique, les publications de sensibilisation et d'information, les événements scientifiques (essentiellement en France) ainsi que la dimension internationale.

Environmental policies and ecology : fifty years in review

The main events making out fifty years of environmental policy are recorded in a synoptic table. French législatives clauses and the state organisms set up to enforce them are jointly noted. Bcsides, it has seemed interesting to list a larger range of events, mostly French, but also Worldwide, concerning the development of the ecologist movcment, newspapers, books, publications, and the rclated scientific events.

\section{INDEX}

Mots-clés : Politique, environnement, écologie

Keywords : Ecology, environment, politics

\section{AUTEUR}

\section{MARIE CLAUDE GUERRINI}

Chargée de recherches au CNRS, elle travaille sur l'évolution du milieu rural et plus particulièrement sur l'analyse de l'interface Homme/Milieu Naturel. Elle a notamment collaboré au livre Du rural à l'environnement. La question de la Nature aujourd'hui, N. Mathieu, M. Jollivet (Dir.), (L'Harmattan, 1989). 